\title{
Editorial
}

\section{A focus on vulnerable road users}

\section{Ralf Risser, Karel Pospíšl, Matúš Šucha}

This journal issue - the first one of the TOTS journal in its "new clothes" - contains several articles that in one or the other way deal with vulnerable road users. The association ICTCT was asked by the University of Technology of Beijing, one of its members, to organise a workshop about how to develop a strategy to promote cycling in China. At the same time plans had developed to reinstall the journal TOTS. The responsible of the journal some of whom were involved in this workshop had the idea to make the first issue a special one by offering those who gave presentations at the workshop to be prioritised in case they planned to submit an article about their presentation. A closer look at the title of the Beijing workshop - referring to a strategy to promote cycling - makes one think of the concept of marketing, or social marketing. It is the explicit goal of marketing to promote something. Social marketing connected to mobility issues would e.g. deal with the promotion of special types of mobility-mode choice. It would for instance deal with the question how to influence mode choice of any target audience in such a way that improvements of the quality of life of individuals and their society become tangible. It would help to identify the reasons, why people resist change of their present mobility habits and uncover affordable benefits of a change of choices, which the audience cares about. Based on these findings better preconditions for certain mode choices would be developed and the advantages of those would be communicated in a compelling and cost-effective way.

The use of social marketing has been proven to be effective in many social programs: from reducing smoking rates, increasing immunisation rates among children, increasing physical activity in elderly change nutrition habits in the population etc. The concepts, theories and design components for nutrition education and enhancing physical activity among older persons have been well described and everything points in the direction that mode-choice can be influenced effectively and efficiently with the help of social-marketing measures. Social marketing is characterised by the 5 Ps displayed in figure 1 .

The development of informative and persuasive communication ( $\mathrm{P}=$ Promotion) ensures that the "product" (e.g. preconditions for a certain mode

\section{PRODUCT}

Program developed regarding needs and interests of target population. Appreciated physical (e.g. design) and organisational characteristics to be introduced or pointed out

\section{PLACEMENT}

Logistics involved in obtaining necessary services and actions \& identifying and involving community leaders, organisations, associations, mediators, providers of education of the target group population, models etc.

\section{PRICE}

Costs and benefits of a change in the envisaged direction - e.g. from car to bicycle - in money, time, effort. Issues of behavior change: benefits, profits vs. barriers, costs

\section{PERSONS' CHARACTERISTICS (attitudes, habits etc.)}
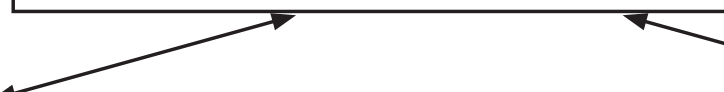

\section{PROMOTION}

Practical strategies and means for making the goals for a change and the expected results of such change visible and attractive to target groups \& advertising (announcements) and events in the media and via involved organizations (including researchers) 
choice) is presented in an appropriate manner. This includes formulating the objectives of mode-change efforts, addressing the appropriate persons and institutions in an appropriate way ( $\mathrm{P}=$ Placement), and tracking responses to all efforts in order to adapt offers ( $\mathrm{P}=$ Product) and arguments to the users' needs and expectations. It takes into account what the audience wants, needs, and expects, i.e. their satisfaction/ dissatisfactions. This means that social marketing has to consider the attitudes and habits of the target audience ( $\mathrm{P}=$ Persons' characteristics), their sociodemographic and psychological characteristics, their socialisation, and benefits and barriers as perceived by them $(\mathrm{P}=$ Price $)$.

When looking at the papers included in the first issue of TOTS it is easy to see that only parts of the aspects that should be covered according to the social marketing approach are taken up explicitly, there. Several articles deal with the Product part; Charlotte Tønning and Niels Agerholm deal with young drivers and effects from graduated driving license. Kaufmann and Vankov address the same group, their focus is on motivating activities and campaign work in different countries in- and outside Europe. On a first glance this has nothing to do with promoting cycling at all. However, when using the marketing idea as a frame, one could say that good driver education must include lessons about what car drivers have to consider with respect to vulnerable road users. If education is effective, i.e. if it shapes the behaviour of trainees in the wished-for way, but if at the same time taking care of vulnerable road users is not a topic dealt with, then education is effective but it has severe short comings. Tønning and Agerholm do not take up this point explicitly, while Christine Chaloupka-Risser and Elisabeth Füssl do so in their paper about Communication of bicyclists with car drivers and safety critical events. They assume that better communication with car drivers will make the life of bicyclists easier most of all by understanding what in this communication is safety critical. Based on this, in a later step, safety critical events can be mitigate or eliminated, thus improving the Product and making cycling easier by reducing the Price (= the hardships one has to accept when cycling).

In his paper about Bicycle traffic in the Czech Republic: the ways of influencing the behaviour of people involved in it Matus Sucha takes refers indirectly to some aspects that are part of the Promotion and Price areas, and he takes explicitly up Persons' characteristics as described above. Combining the findings from these areas is a precondition to develop preconditions for cycling - the Product - further in such a way that these offers would be accepted by (greater parts of) the population.

The approach of Ralf Risser in his article Mobility and comfort of vulnerable road users is similar. He discusses what could improve the preconditions for pedestrians and cyclists, with special consideration of ITS - Intelligent Transport Systems - that could be deployed to the advantage of vulnerable road users. In his case the question how vulnerable road users look at such potential advantages themselves is even dealt with empirically, by carrying out focus-group interviews with vulnerable road users in different $\mathrm{Eu}$ ropean countries.

Finally, Benjamin Schreck reports about Cycling and designing for cyclists in Germany. There he provides an overview of road safety date, research carried out and existing guidelines. Thereby, the assumption that all activities referred to have the effect to promote cycling is implicit, and it is an assumption that is based on the view of experts on the topic. 